\title{
A CONCEPTUAL FRAMEWORK FOR BUILDING CIM-BASED ONTOLOGIES
}

\author{
Emmanuel Lavinal, Thierry Desprats and Yves Raynaud \\ IRIT-UPS, UMR 5505 CNRS. 118 Route de Narbonne, F-31062 Toulouse Cedex 4, France \\ \{lavinal, desprats, raynaud\}@irit.fr
}

\begin{abstract}
Cooperative Management has appeared as a promising paradigm on which Network and System Management (NSM) solutions should be partially or entirely based. This paper proposes a conceptual framework to obtain, from any CIM schemas, NSM frame-based ontologies which can then be used in cooperative management solutions.
\end{abstract}

Key words: Information models, cooperative management, CIM, ontology

\section{INTRODUCTION}

From a technological viewpoint, Multi-Agents Systems (MAS) constitute the natural candidate to support the implementation of this cooperative management paradigm $[1,2]$. In such a context, autonomous entities interact in a cooperative way to reach a common objective of NSM. This implies that the involved entities understand each other and share a common representation of the domain knowledge. More precisely, these entities interact by exchanging primitives specified by an Agent Communication Language (ACL) like FIPA-ACL. These messages, called performatives, convey information about illocutionary power of the interaction within the dialogue (e.g. assertion, question, order, etc.); this information, devoted to protocol aspects, is totally independent from the application domain. Another conveyed information is directly related to the domain because it defines the real semantics of the message. A shared vocabulary is required to represent the "universe of discourse" by specifying the common concepts, attributes and relationships which describe the target application domain. This vocabulary is called an ontology and is used by any agent to express its knowledge about its environment [3, 4].

If we want to promote the use of MAS in the NSM domain, having ontologies related to this domain is absolutely necessary. As there are none existing at the moment, our objective is to provide a generic and automatic way to obtain NSM

The original version of this chapter was revised: The copyright line was incorrect. This has been corrected. The Erratum to this chapter is available at DOI: 10.1007/978-0-387-35674-7_66 
ontologies which then should be used in a cooperative NSM context. Rather than trying to specify one NSM ontology able to cover all the needs in this field, our idea was to benefit from the very important works done by the DMTF: the CIM effort currently appears as the most successful and recognized approach.

The conceptual framework we propose is based on a precise mapping which can be applied to build NSM ontologies from existing CIM schemas. We show how it is possible to express the CIM Meta Schema thanks to the OKBC Knowledge Model, and therefore, how to obtain frame-based NSM ontologies from any CIM schema.

\section{OKBC AND CIM OVERVIEW}

OKBC (Open Knowledge Base Connectivity) [5] is a standard for frame-based ontology. It specifies a knowledge model (KM) which provides a set of constructs commonly found in frame-based knowledge representation systems. It includes frames, classes, slots and facets. A frame is a primitive object that represents an entity in the domain of discourse. A frame that represents a class is called a class frame, a frame that represents a slot is called a slot frame, etc. Basically, classes are similar to object-oriented approaches, slots describe properties or attributes of classes and facets describe properties of slots. Protégé-2000 [6] is an OKBCcompatible ontology editing and knowledge acquisition environment. The use of metaclasses makes Protégé-2000 easily extensible and enables its use with other knowledge models. Due to its capacity to be expressed in a formal language like $\mathrm{KIF}$ [7] and to its numerous implementations, we selected OKBC as the target environment to obtain NSM CIM-based ontologies.

CIM [8] is a conceptual information model for describing management that is not bound to a particular implementation. CIM defines a collection of schemas which provides the actual model descriptions. The CIM Schema is structured into three distinct layers: the core model, the common models and the extension models. These generic schemas constitute a very important work for vendors who can model their management information on a common and standard basis. CIM also specifies a meta schema which defines the terms used to express the model and its usage and semantics. Some elements of the meta model (MM) are descended from objectoriented approaches (Classes, Properties, Methods, Associations, References) and others descend from management or data base domains (Qualifier, Trigger, Indication).

\section{THE CONCEPTUAL FRAMEWORK}

On the one hand, ontologies and CIM serve very different purposes: ontologies allow sharing and reuse of knowledge to provide a common understanding of some domain whereas CIM modelizes management information to provide integrity constraints for management systems. On the other hand, ontologies and CIM have some common points: they both provide vocabulary and structure for describing 
particular information and they also have similar modeling levels (Figure 1). It is therefore legitimate to compare both. We chose the highest level of abstraction to perform our mapping (meta model level), that is between the OKBC knowledge model and the CIM meta schema. This choice is motivated by the fact that we wanted to provide a high level of genericity to exploit this work in all the underlying levels, allowing therefore the construction of ontologies from any CIM model.

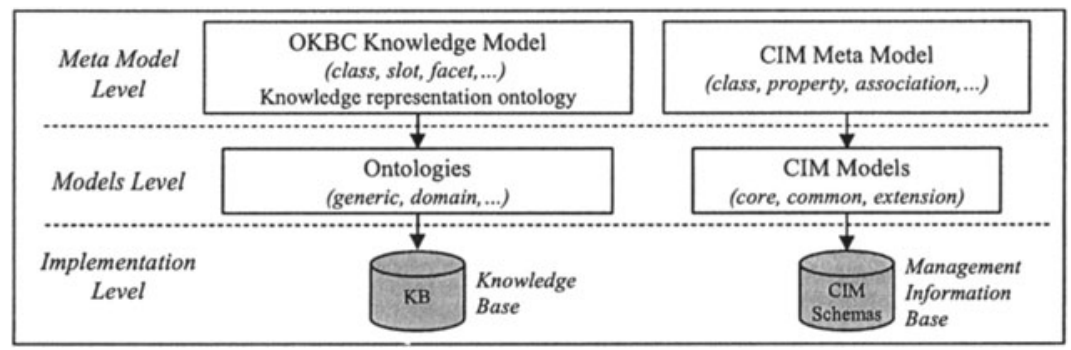

Figure 1. OKBC vs. CIM modeling levels

We first made a comparative analysis between the data types, concepts and standard elements defined in the two approaches, and then we established a set of precise correspondences at a modeling level to reconcile the differences. We will briefly expose our work on the concepts.

We identified three categories of concepts: $(i)$ concepts that are exactly the same, (ii) concepts that are the same but expressed in a different way and finally (iii) concepts of the CIM meta model that do not have an equivalent in the OKBC knowledge model. These categories were concealed either by direct equivalences or by the extension of the OKBC knowledge model to express the new CIM meta model concepts. For example, classes, slots as properties and inheritance are concepts exactly the same in the two approaches. Concerning category (ii), we can quote the concept of association that can be represented as a special slot in OKBC whereas CIM defines an association as a class. Therefore we created a metaclass CIM:ASSOCIATION to respect the CIM specification. Finally, the concepts of qualifier, method, trigger, etc. fall in the category (iii): we defined new metaclasses and metaslots that we configured to reflect exactly the CIM concepts.

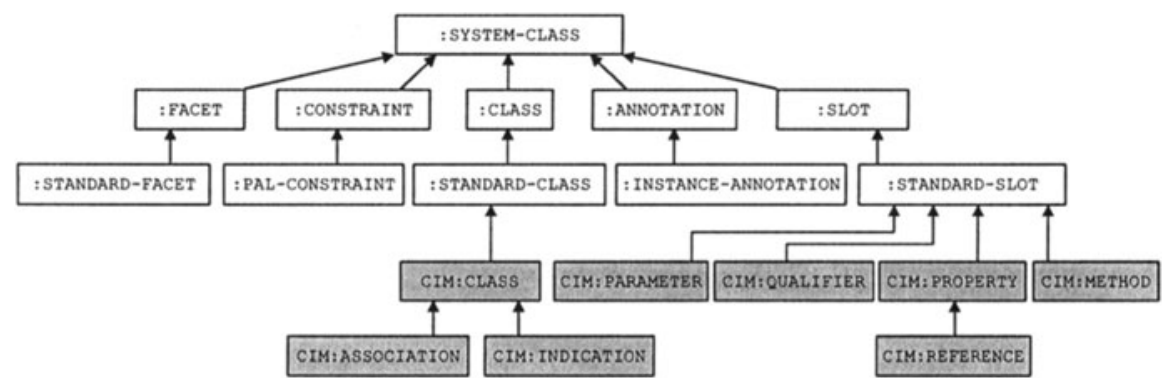

Figure 2. Specialization of the KM of Protégé-2000 to express the MM of CIM 
The result of this work is a complete expression of the meta model of CIM in the OKBC knowledge model. We applied this work in the environment Protégé-2000, adding metaclasses and metaslots to the system architecture. Figure 2 illustrates the Protégé knowledge model extended by the new concepts descended from CIM.

\section{CONCLUSION AND FUTURE WORKS}

The presented work allows us to express the CIM Meta Schema as a framebased ontology. This has been achieved by extending the OKBC knowledge model in the Protégé-2000 environment in order to specify all the concepts proper to CIM. We also realised a full mapping concerning both the data types and the CIM standards qualifiers. This precise and complete projection has been done at the respective "Meta" levels.

Based on that extended knowledge model, we have obtained the expression of the entire standard CIM "Network" schema as a Protégé-2000 ontology. This result shows that the mapping defined at the "Meta" level may constitute a solid basis to obtain NSM-related ontologies from any existing CIM models. Both the Core and Common CIM schemas defined by the DMTF may be expressed in such a way as ontologies. In the same manner, all the CIM extension schemas which have been (or will be) defined by all the categories of CIM users may be transformed into ontologies. By providing a "Meta-mapping" solution, we have reached a high level of genericity to easily reuse the whole efforts made around the CIM modeling approach: any CIM-based model can become a NSM frame-based ontology.

Current works intend to automate the mapping process. Based on our proposal, a tool which allows to generate a Protegé-2000 ontology from the analysis of a CIMXML file (describing CIM schema) is under construction. This tool will help to integrate CIM models as ontologies within cooperative NSM solutions without requiring any expertise on ontologies.

\section{REFERENCES}

[1] J.P. Martin-Flatin, S. Znaty. Two Taxonomies of Distributed NSM Paradigms, Emerging Trends and Challenges in Network Management, Chapter 3, June 2000

[2] K. Boudaoud, Z. Guessoum, C. Mc Cathie Neville, P. Dubois. Policy-based Security Management Using a Multi-Agent System, Workshop HPOVUA, Berlin, June 2001

[3] A. Gómez-Pérez. Ontological Engineering: a State of The Art, Expert Update, 1999

[4] C. van Aart, R. Pels, G. Caire, F. Bergenti. Creating and Using Ontologies in Agent Communication, Workshop on Ontologies in Agent Systems, Italy, July 16, 2002

[5] V.K. Chaudhri \& al. Open Knowledge Base Connectivity 2.0.3, April 1998

[6] N.F. Noy, R.W. Fergerson, M.A. Musen. The knowledge model of Protégé-2000: combining interoperability and flexibility, EKAW'2000, France, October 2-6, 2000

[7] Knowledge Interchange Format. Draft proposed American National Standard: http://logic.stanford.edu/kif/dpans.html

[8] Common Information Model Specification. DMTF, http://www.dmtf.org/standards/ 